\title{
Prognostic Factors of IVF\&ICSI Cycle Cancellation in Patients with Endometriosis-Related Infertility
}

\author{
Elif KULAHCI ASLAN', Kiper ASLAN', Cihan CAKIR², Isil KASAPOGLU1, Berrin AVCI², Baris ATA³, \\ Gurkan UNCU1
}

Bursa, Turkey

\section{ABSTRACT}

OBJECTIVE: To elucidate the prognostic factors for intracytoplasmic sperm injection cycle cancellation in patients with endometriosis-related infertility.

STUDY DESIGN: This was a retrospective cohort study and conducted at the Assisted Reproductive Technology center of Uludag University School of Medicine, between the years 2011-2017. The electronic database was screened and infertile patients with endometriosis, without male factor infertility, systemic disease, or undefined adnexal mass, and aged $<40$ were selected. The endometriosis phenotype of all cycles was classified into three subgroups: superficial endometriosis, ovarian endometrioma and deep infiltrating endometriosis. Cycles were divided into two groups: Group I (Cycle Cancellation) vs. Group II (Embryo transferred).

RESULTS: Forty-four cycles were canceled and in 178 cycles, the embryo was able to be transferred. When the groups were compared age and day 3 FSH levels were statistically higher, and anti-Mullerian hormone and antral follicle count were statistically lower in Group I. The presence of adenomyosis was higher in Group I (64\% vs. $40 \%$ p<0.01). The surgery rate with laparotomy was higher in Group I $(54.5 \%$ vs. $13.5 \% p<0.01)$. Antral follicle count remained as the only independent factor associated with prognoses of the IVF cycle with binary logistic regression analysis. Cancellation rates were similar between the phenotypes of endometriosis.

CONCLUSIONS: Poor ovarian reserve, advanced age, presence of adenomyosis, and history of laparotomy are negative prognostic factors associated with intracytoplasmic sperm injection cycle cancellation in endometriosis-related infertility. Antral follicle count is the only independent factor in predicting cycle cancellation. The phenotype of endometriosis does not affect the results.

Keywords: Adenomyosis, Cycle cancellation, Endometriosis, Infertility, Intracytoplasmic sperm injections

Gynecol Obstet Reprod Med. 2022;28(1):44-49

1 Uludag University School of Medicine Department of Obstetrics \&
Gynecology Bursa, Turkey
2 Uludag University School of Medicine Department of Histology \&
Embryology Bursa, Turkey
3 Koc University School of Medicine Department of Obstetrics \& Gynecology
Istanbul, Turkey
Address of Correspondence: Kiper Aslan
Uludag University School of Medicine
Department of Obstetrics \& Gynecology,
16059 Gorukle, Bursa Turkey
kiperaslan@yahoo.com.tr

How to cite this article: Kulahci Aslan E. Aslan K. Cakir C. Kasapoglu I. Avci B. Ata B. Uncu G. Prognostic Factors of IVF\&ICSI Cycle Cancellation in Patients with Endometriosis-Related Infertility. Gynecol Obstet Reprod Med. 2022;28(1):44-49

\section{Introduction}

Endometriosis is an inflammatory disorder and defined as the presence of endometrial-like tissue outside of the uterus, and its estimated prevalence is $10-15 \%$ in reproductive women (1). It mainly induces infertility, and the prevalence of endometriosis rises above $40 \%$ in infertile women since endometriosis distorts pelvic anatomy, impaired peritoneal function, altered implantation and decreased ovarian reserve (2-6).

Treatment of endometriosis-related infertility is based on surgery, assisted reproductive technology (ART), or both interventions together. Assisted reproductive technologies may not be effective in some cases with endometriosis-related infertility since all these factors listed above make the treatment more difficult. Intracytoplasmic sperm injection (ICSI) cycles are frequently canceled because of insufficient ovarian response to the stimulation, a decreased number or quality of oocytes (7), decreased fertilization rate, or maturation arrest. In a study reported by Kuroda, it was found that the cancellation rates of cycles (including cases of empty follicles, degen- 
erating and unfertilized eggs) were $35.5 \%$ in patients with unoperated endometrioma, $47.1 \%$ in operated endometrioma, $40 \%$ in endometriosis with no endometrioma, and $14.8 \%$ in couples with tubal factor infertility (8). Bongioanni et al. also found that the cancellation rate was $7.5 \%$ in unoperated endometrioma, $9.8 \%$ in operated endometrioma, and $2.9 \%$ in tubal factor infertility and they commented that the cancellation rates were higher in endometriosis-related infertility (9). The reason for these high cancellation rates remains unclear. There is no study about which factors affect the cancellation rate in endometriosis-related infertility.

In this study, we aimed to elucidate the factors affecting the cancellation of the cycle, depending on the phenotypes of endometriosis and other characteristics of the cycles.

\section{Material and Method}

This retrospective cohort study was conducted at the ART center of Uludag University School of Medicine. The research ethics committee of Uludag University approved the study protocol (2017-1/52). The electronic database between Jan 2012/Jan 2017 was screened to identify patients with endometriosis.

Women aged $>40$ years, with chronic systemic diseases (diabetes mellitus, hypertension, etc.), and with suspicion of malignancy, and couples with male infertility were excluded from the study. The enrolled patients' ICSI cycles were compared in three endometriosis phenotypes: superficial endometriosis, ovarian endometrioma, and deep infiltrating endometriosis. A patient who had two phenotypes of endometriosis was enrolled in the more severe group. Superficial endometriosis was diagnosed during laparoscopic surgery, which was performed for other indications such as tubal factor infertility, unexplained infertility, etc. It is defined as the presence of endometriotic lesions on the peritoneum. Ovarian endometrioma is defined as homogenous, usually unilocular, focal lesions in the ovary with low-level echoes in ultrasound imaging and with a shading sign in magnetic resonance imaging (10). Deep infiltrating endometriosis was diagnosed by bimanual pelvic examination (palpable rectovaginal endometriotic nodule), by transvaginal ultrasound screening and MRI, or it was defined based on the visual diagnosis of an infiltrating type of endometriosis in surgeries $(11,12)$.

Adenomyosis was defined as ingrowths of the endometrial cells into the myometrium. Patients were screened by transvaginal ultrasound and the presence of two of the listed criteria was defined as adenomyosis. 1-No distinction of the endometrial-myometrial junction; 2-Asymmetry of the anterior and posterior myometrium; 3-Subendometrial myometrial striations; 4-Myometrial cysts and fibrosis; and 5Heterogeneous myometrial echotexture (13).

All patients underwent ovarian stimulation by using either ultra-long protocol, long protocol, antagonist protocol, microdose flare-up protocols, or natural cycles. Goserelin acetate $3.6 \mathrm{mg}$ monthly depot form was injected for 3 months, or daily leuprolide acetate $0.1 \mathrm{mg} /$ daily injection was applied for 3 months for pituitary desensitization in the ultra-long protocol. Daily injections of leuprolide acetate were started on the $21 \mathrm{st}$ day of the cycle in long protocol. After pituitary desensitization, daily gonadotropin injection with 150-600 IU/day was started and daily antagonist (cetrorelix-ganirelix $0.25 \mathrm{mg}$ ) injections were applied after reaching 12-14 mm follicle in short antagonist protocol. Trigger (recombinant human chorionic gonadotropin (hCG) or leuprolide acetate) was given when $>3$ follicles greater than $17 \mathrm{~mm}$. Oocyte retrieval was done 34-36 hours after hCG injection. The luteal phase was supported with vaginal $8 \%$ progesterone gel twice a day, starting on the evening of oocyte retrieval and continued until a negative pregnancy test or detection of fetal cardiac activity. The day of embryo transfer was decided based on the number of available embryos, and the embryo qualities.

Three types of cycle cancellation were defined.

Type A is identified as no follicular development despite the maximum dose of gonadotropin injections.

Type B is identified as a full fertilization failure.

Type $\mathrm{C}$ is identified as a maturation arrest and defined whenever the maturation of the embryo stops.

All cycles were classified into two groups. The first group (Group I) consisted of canceled cycles, and the second group (Group II) consisted of embryo transferred cycles. Both groups were divided into three subgroups depending on the phenotype of endometriosis. Factors associated with a cycle cancellation which depended on the phenotype of the disease were found. Independent risk factors associated with cycle cancellation were investigated further by using logistic regression analysis, and also the effects of the presence of adenomyosis and laparotomy history on ovarian reserve were analyzed.

The Kolmogorov-Smirnov test was used for assessing whether the variables followed a normal distribution or not. Variables were reported as mean \pm standard and median (minimum-maximum) deviation values. Student $T$-test or Mann Whitney U test was used depending on the distribution of variables. According to the normality test results, independent samples $t$-test was used for between-group comparisons. Categorical variables were reported as n (\%) and compared by Pearson Chi-square test or Fisher-Freeman-Halton-Test. To determine the independent risk factors that affect IVF cycle cancellation, binary logistic regression analysis with backward selection was performed. SPSS (IBM Corp. Released 2012. IBM SPSS Statistics for Windows, Version 21.0. Armonk, NY: IBM Corp.) was used for performing statistical analysis and the level of significance was set at $\alpha=0.05$. 


\section{Results}

Between the years Jan 2012-Jan 2017, there were 2515 cycles. All cycles were screened and 222 of the cycles were enrolled in the study.

Forty-four (19.8\%) cycles were canceled and the embryo was able to be transferred in $178(80.2 \%)$ cycles.

Four $(9.1 \%)$ cycles were canceled due to inefficient response (Type A), 24 (54.6\%) cycles were canceled due to fertilization failure (Type B) and $16(36.3 \%)$ cycles were canceled due to maturation arrest (Type $\mathrm{C}$ ).

When the groups were compared, age was statistically higher in Group I (34 (26-43) yrs. vs. 30 (8-40) yrs. $p<0.01)$.

Day 3 FSH levels were statistically higher in Group I (8.1 $(2.6-23.2) \mathrm{IU} / \mathrm{dL}$ vs. $6.2(2.6-21.8) \mathrm{IU} / \mathrm{dL}$, respectively $p<0.01)$.

AMH level was statistically lower in Group I (0.5 (0.0110) $\mathrm{ng} / \mathrm{mL}$ vs. $1.9(0.01-10) \mathrm{ng} / \mathrm{mL} p<0.01)$.
AFC was statistically lower in Group I (4 (1-16) vs. 8 (120) $p<0.01)$.

The presence of adenomyosis was higher in Group I (64\% vs. $40 \% p<0.01)$.

The rate of surgery with laparotomy was higher in Group I $(54.5 \%$ vs. $13.5 \% p<0.01)$ (Table I).

Statistically significantly different factors $(p<0.05)$ were further analyzed with binary logistic regression analysis to determine the independent prognostic factors for cycle cancellation. Antral follicle count (OR:0.81, CI:0.70:0.93) remained as the only independent factor associated with prognoses of IVF cycles (Table II).

Cycle cancellation rates were $16.6 \%$ in patients with superficial endometriosis, $19.7 \%$ in patients with ovarian endometrioma, and $21.2 \%$ in patients with deep infiltrating endometriosis, and there were no statistically significant differences between the phenotypes of endometriosis (Table III).

Table I: Cancelled cycles (group I) vs. transferred cycles (group II) - demographic data

\begin{tabular}{|c|c|c|c|}
\hline & $\begin{array}{l}\text { Group I } \\
(n=44)\end{array}$ & $\begin{array}{l}\text { Group II } \\
(n=178)\end{array}$ & $p$ \\
\hline Age & $34(26-40)$ & $30(8-40)$ & $<0.01$ \\
\hline BMI $\left(\mathrm{kg} / \mathrm{m}^{2}\right)$ & $23.4(18.9-36.1)$ & $23.3(16-37)$ & 0.313 \\
\hline Gravida & $0(0-2)$ & $0(0-4)$ & 0.729 \\
\hline Duration of infertility (yrs) & $5(1-18)$ & $4(1-16)$ & 0.754 \\
\hline Day 3 FSH (IU/dL) & $8.1(2.6-23.2)$ & $6.2(2.6-21.8)$ & $<0.01$ \\
\hline Day 3 Estradiol (pg/mL) & $46.1(5.4-240)$ & $42(1.5-310)$ & 0.07 \\
\hline $\mathrm{AMH}(\mathrm{ng} / \mathrm{mL})$ & $0.5(0.01-10)$ & $1.9(0.01-10)$ & $<0.01$ \\
\hline $\operatorname{AFC}(n)$ & $4(1-16)$ & $8(1-20)$ & $<0.01$ \\
\hline Adenomyosis (\%) & $28 / 44(64 \%)$ & $72 / 178(40 \%)$ & $<0.01$ \\
\hline Surgery (\%) & $22 / 44(50 \%)$ & $104 / 178(58 \%)$ & 0.255 \\
\hline Surgery: & & & $<0.01$ \\
\hline Laparoscopy & $10 / 22(45.5 \%)$ & $90 / 104(86.5 \%)$ & \\
\hline Laparotomy & $12 / 22(54.5 \%)$ & $14 / 104(13.5 \%)$ & \\
\hline \multicolumn{4}{|l|}{ Endometriosis Phenotype: } \\
\hline Superficial & $3 / 44 \quad(6.8 \%)$ & $15 / 178(8.4 \%)$ & \\
\hline Endometrioma & $31 / 44(70.5 \%)$ & $126 / 178(70.8 \%)$ & 0.916 \\
\hline Deep infiltrating & $10 / 44(22.7 \%)$ & $37 / 178(20.8 \%)$ & \\
\hline
\end{tabular}

Note: Values are medians with minimum and maximum

Table II: Significant prognostic factors of cycle cancellation after logistic regression analysis

\begin{tabular}{|c|c|c|c|}
\hline Factors & Wald & OR $(95 \% \mathrm{CI})$ & $p$ \\
\hline AFC & 8.70 & $0.81(0.70: 0.93)$ & 0.003 \\
\hline \multicolumn{4}{|c|}{ Model $X^{2=16.55 ; p<0.001}$} \\
\hline \multicolumn{4}{|c|}{ Pseudo $\mathrm{R}^{2}=0.16$} \\
\hline$n=222$ & & & \\
\hline
\end{tabular}

OR: Odds ratio, Cl: Confidence interval 
Table III: Cycle cancellation-depending on phenotypes of endometriosis

\begin{tabular}{|c|c|c|c|c|c|}
\hline & Superficial & Endometrioma & Deep Infiltrating & Total & $p$ \\
\hline No. of cancelled cycles & $3 / 18(16.6 \%)$ & $31 / 157(19.7 \%)$ & $10 / 47(21.2 \%)$ & $44 / 222$ & $\begin{array}{l}0.916^{*} \\
19.8 \%\end{array}$ \\
\hline $\begin{array}{l}\text { Inefficient } \\
\text { response }\end{array}$ & $0 / 18$ & $1 / 157(0.6 \%)$ & $3 / 47(6.3 \%)$ & $\begin{array}{l}4 / 44 \\
9 \%\end{array}$ & $0.147^{* *}$ \\
\hline $\begin{array}{l}\text { Fertilization } \\
\text { Failure }\end{array}$ & $2 / 18(11.1 \%)$ & $17 / 157$ (10.8\%) & $5 / 47(10.6 \%)$ & $\begin{array}{l}24 / 44 \\
54 \%\end{array}$ & \\
\hline $\begin{array}{l}\text { Maturation } \\
\text { Arrest }\end{array}$ & $1(5.5 \%)$ & $13 / 157(8.2 \%)$ & $2 / 47(4.2 \%)$ & $\begin{array}{l}16 / 44 \\
36 \%\end{array}$ & \\
\hline
\end{tabular}

*Kruskal-Wallis Test, ${ }^{* *}$ Fisher-Freeman-Halton Test.

\section{Discussion}

It is widely known that endometriosis has a challenging impact on infertility treatment. Both couples and doctors start the infertility treatment with high hopes, but our results show that $20 \%$ of couples with endometriosis-related infertility are defeated in this battle because of cycle cancellation.

In this study, we aimed to elucidate the prognostic factor for ICSI cycle cancellation in patients with endometriosis-related infertility and to find out whether the phenotype of endometriosis affects the cycle cancellation rate. Our findings indicate that only ovarian reserve markers, particularly AFC, have prognostic value in predicting the risk of canceling the cycle.

The cycle cancellation rate of all endometriosis-related infertility cycles was $19.8 \%$ in our study. There is a limited number of studies that inform about cycle cancellation rates of ERI. All these studies have different cycle cancellation rates. The cycle cancellation rates of the studies in the literature vary between $7.5 \%$ and $47.1 \%(8,9,14)$. The variable cycle cancellation rates between studies may be related to the heterogeneity of patient distribution, and the stage or phenotype of the disease. Thus, we further analyzed the cycle cancellation rates of the phenotypes of endometriosis. However, it seems that the phenotype of endometriosis is not related to cycle cancellation. According to our results, four prognostic factors are related to cycle cancellation. These factors are age, ovarian reserve (day $3 \mathrm{FSH}, \mathrm{AMH}$, and $\mathrm{AFC}$ ), presence of adenomyosis, and laparotomy history. With logistic regression analysis, antral follicle count was found to be the only independent factor related to cycle cancellation.

It is widely known that advanced age negatively affects fertility. As age increases, fecundity decreases. The fecundity of women decreases gradually after 32 years; however, a further significant decrease occurs after 37 years (15). The reason for age-related decrease in fertility maybe because of altered ovarian reserve and metabolism. In a prospective study about women's age and ovarian reserve, altered follicular metabolism was found in women with either reduced ovarian reserve or advanced maternal age (16). The presence of endometriosis also has a detrimental effect on ovarian reserve by itself. The ERROR (Endometrioma Related Reduction in Ovarian Reserve) study showed that women with endometrioma experience a progressive decline in serum AMH level, which is faster than that in healthy women. Thus, both presence of endometriosis and advanced age may have a negative impact on fertility. This may be related to cycle cancellation. In our study, we found a mean women's age of 34 (26-43) in Group I and of 30 (8-40) in Group II. According to our results, women's age is one of the prognostic factors for predicting the risk of cycle cancellation.

We found increased day 3 FSH and AMH levels and decreased AFC levels in Group I. All these results are related to decreased ovarian reserve. With logistic regression analysis, the antral follicle count was found to be the only independent factor related to cycle cancellation. These findings indicate that decreased ovarian reserve, especially decreased AFC, is related to a high possibility of cycle cancellation independent of endometriosis subtypes. In a study about poor ovarian response and cycle cancellation according to AMH levels during different days of the menstrual cycle, results indicated that a day $3 \mathrm{AMH}$ level below $0.3 \mathrm{ng} / \mathrm{ml}$ predicted a high risk of cycle cancellation with $70 \%$ sensitivity and $90 \%$ specificity. In our study, we found an AMH level of $0.5(0.01-10) \mathrm{ng} / \mathrm{mL}$ in Group I (It was $1.9(0.01-10) \mathrm{ng} / \mathrm{ml}$ in Group II). It is known that AFC is a good predictor of ovarian response even in an ovary with endometrioma. In our study, the antral follicle count was found to be the only independent prognostic factor related to cycle cancellation (OR:0.81, CI:0.70:0.93). This result shows the importance of AFC for assessing ovarian reserve and predicting cycle cancellation. In the ERROR study, endometrioma was found to be associated with a greater progressive decline in ovarian reserve than that in normal healthy women (17). Another study conducted in our department was related to oocyte quality in endometriosis. In that study, patients were divided into two groups, namely male factor and endometriosis. Of the abnormal oocytes determined in all cycles, $59.1 \%$ of oocytes were in the endometriosis group and $40.9 \%$ of oocytes in the malefactor group. This difference was statistically significant and showed that endometriosis negatively affected the oocyte morphology. 
In this study, a new classification was formed for cycle cancellation. Type A: inefficient ovarian response, Type B: fertilization failure, and Type $\mathrm{C}$ : maturation arrest. The cycle cancellation rate was $9.1 \%$ for Type A, $54.6 \%$ for Type B, and $36.3 \%$ for Type $\mathrm{C}$. The main reason for cancellation is the fertilization problem, with $54 \%$. The results show that the presence of endometriosis negatively affects ovarian quality while causing a decrease in ovarian reserve. Furthermore, if a patient with endometriosis has poor ovarian reserve, the risk of cycle cancellation is higher than in the ones with normal ovarian reserve independently of the subtypes of endometriosis.

Another prognostic factor is the presence of a history of laparotomy. The laparotomy rate was $54.5 \%$ in Group I and $13.5 \%$ in Group II $(p<0.01)$, and this difference were statistically significant. There is an ongoing debate as to whether laparotomy decreases ovarian reserve. There are studies about surgery in endometriosis, and the results of these indicate that laparoscopy may have more detrimental effects on ovarian reserve than laparotomy (18). However adversely, our results indicated that the cycle cancellation rate was higher in the patients who were operated by laparotomy than those operated by laparoscopy. We did not know the ovarian reserve of the patients before surgery so that we could not interpret the effect of the surgery type on ovarian reserve. Furthermore, we did not know whether stripping or coagulation was used during the endometrioma excision. As a result, we may conclude that (excluding contributing factors) the presence of laparotomy history has a negative impact on cycle success.

The other issue concerning cancellation is the presence of adenomyosis. We found an adenomyosis rate of $64 \%(28 / 44)$ in Group I vs. 40\% (16/44) in Group II and this difference was statistically significant. Nevertheless, it was found that the presence of adenomyosis is not an independent marker of cycle cancellation in the univariate analysis. Although it is not an independent factor in terms of cancellation, we can say that the presence of adenomyosis is essential for determining the cycle cancellation.

Besides all these factors discussed above, we also analyzed whether the endometriosis phenotype is predictive for cycle cancellation. Our cycle cancellation results were comparable: $16.6 \%$ in superficial endometriosis, $19.7 \%$ in ovarian endometrioma, and $21.2 \%$ in deep infiltrating endometriosis groups. It was found that the cycle cancellation is mainly based on ovarian functions and that the endometriosis phenotype does not affect the cycle cancellation rates.

The risk of cancellation can be predicted by analyzing ovarian reserve parameters carefully. Day $3 \mathrm{FSH}, \mathrm{AMH}$, and AFC are important parameters to predict cycle success in endometriosis-related infertility. Antral follicle count is the only independent factor in predicting cycle success. The results can be confirmed with further larger-scale studies.
Acknowledgments: We thank all Stuff of Uludag University Faculty of Medicine ART Center for their support.

The study was conducted in accordance with the Declaration of Helsinki

Funding: There was no funding for this study. Conflict of Interest: There is no conflict of interest in this study.

Contribution to the paper: There is no funding.

Contribution to the paper: There is no conflict of interest. Author contribution: EKA, KA: Data collection, statically analysis, writing the paper. CC, BA: Embryology laboratory. IK: Folliculometry, oocyte pick up. BA, GU: Writing and reviewing the paper

\section{References}

1. Mowers EL, Lim CS, Skinner B, Mahnert N, Kamdar N, Morgan DM, et al. Prevalence of Endometriosis During Abdominal or Laparoscopic Hysterectomy for Chronic Pelvic Pain. Obstet Gynecol. 127(6):1045-53. PMID: 27159755, Doi: 10.1097/AOG.0000000000001422.

2. Eskenazi B, Warner ML. Epidemiology of endometriosis. Obstet Gynecol Clin North Am. 1997;24(2):235-58. PMID: 9163765, Doi: 10.1016/s0889-8545(05)70302-8.

3. Lebovic DI, Mueller MD, Taylor RN. Immunobiology of endometriosis. Fertil Steril. 2001;75(1):1-10. PMID: 11163805, Doi: 10.1016/S0015-0282(00)01630-7.

4. Kao LC, Germeyer A, Tulac S, Lobo S, Yang JP, Taylor $\mathrm{RN}$, et al. Expression profiling of endometrium from women with endometriosis reveals candidate genes for disease. Based implantation failure and infertility. Endocrinology. 2003;144(7):2870-81. PMID: 12810542, Doi: 10.1210/en.2003-0043.

5. Endometriosis and infertility: A committee opinion. Fertil Steril. 2012;98(3):591-8. PMID: 22704630, Doi: 10.1016/ j.fertnstert.2012.05.031.

6. Uncu G, Kasapoglu I, Ozerkan K, Seyhan A, Oral Yilmaztepe A, Ata B. Prospective assessment of the impact of endometriomas and their removal on ovarian reserve and determinants of the rate of decline in ovarian reserve. Hum Reprod. 2013;28(8):2140-5. PMID: 23624 580, Doi: 10.1093/humrep/det123.

7. Kasapoglu I, Kuspinar G, Saribal S, Turk P, Avc1 B, Uncu G. Detrimental effects of endometriosis on oocyte morphology in intracytoplasmic sperm injection cycles: a retrospective cohort study. Gynecol Endocrinol. 2018;34(3):206-11. PMID: 29057690, Doi: 10.1080/ 09513590.2017.1391203.

8. Kuroda K, Kitade M, Kikuchi I, Kumakiri J, Matsuoka S, Kuroda M, et al. The impact of endometriosis, endometrioma and ovarian cystectomy on assisted reproductive technology. Reprod Med Biol. 2009;8(3):113-8. PMID: 29699316, Doi: 10.1007/s12522-009-0021-1.

9. Bongioanni F, Revelli A, Gennarelli G, Guidetti D, Delle 
Piane LD, Holte J. Ovarian endometriomas and IVF: a retrospective case-control study. Reprod Biol Endocrinol. 2011;9:81. PMID:21679474, Doi: 10.1186/1477-7827-981.

10. Glanc P, Benacerraf B, Bourne T, Brown D, Coleman BG, Crum $\mathrm{C}$, et al. First International Consensus Report on Adnexal Masses: Management Recommendations. J Ultrasound Med. 2017;36(5):849-63. PMID: 28266033, Doi: $10.1002 /$ jum.14197.

11. Woodward PJ, Sohaey R, Mezzetti TP. Endometriosis: radiologic-pathologic correlation. Radiographics. 2001; 21(1):193-216; questionnaire 288-94. PMID: 11158655, Doi: 10.1148/radiographics.21.1.g01ja14193.

12. De Cicco C, Corona R, Schonman R, Mailova K, Ussia A, Koninckx PR. Bowel resection for deep endometriosis: A systematic review. BJOG: Int J Obstet Gynaecol. 2011; 118:285-91. PMID:21040395, Doi: 10.1111/j.14710528.2010.02744.x.

13. Bazot M, Daraï E, Rouger J, Detchev R, Cortez A, Uzan $\mathrm{S}$. Limitations of transvaginal sonography for the diagnosis of adenomyosis, with histopathological correlation. Ultrasound Obstet Gynecol. 2002;20(6):605-11. PMID: 12493051, Doi: 10.1046/j.1469-0705.2002.00852.x

14. Garcia-Velasco JA, Mahutte NG, Corona J, Zúñiga V, Gilés J, Arici A, et al. Removal of endometriomas before in vitro fertilization does not improve fertility outcomes: a matched, case-control study. Fertil Steril. 2004;81(5): 1194-7. PMID:15136074, Doi: 10.1016/j.fertnstert. 2003.04.006.

15. American College of Obstetricians and Gynecologists Committee on Gynecologic Practice and The Practice Committee of the American Society for Reproductive Medicine. Female age-related fertility decline: Committee Opinion No. 589. Fertil Steril. 2014;101(3):633-4. PMID: 24553169, Doi: 10.1097/01.AOG.0000444440.96486.61

16. Pacella L, Zander-Fox DL, Armstrong DT, Lane M. Women with reduced ovarian reserve or advanced maternal age have an altered follicular environment. Fertil Steril. 2012;98(4):986-94.e1-2. PMID: 22818290, Doi: 10.1016/j.fertnstert.2012.06.025.

17. Kasapoglu I, Ata B, Uyaniklar O, Seyhan A, Orhan A, Yildiz Oguz S, et al. Endometrioma-related reduction in ovarian reserve (ERROR): a prospective longitudinal study. Fertil Steril. 2018;110(1):122-7. PMID: 29935810 Doi: 10.1016/j.fertnstert.2018.03.015.

18. Zaitoun MM, Zaitoun MM, El Behery MM. Comparing long term impact on ovarian reserve between laparoscopic ovarian cystectomy and open laparotomy for ovarian endometrioma. J Ovarian Res. 2013;6(1):76. PMID: 24180348, Doi: 10.1186/1757-2215-6-76. 